\title{
The Ponseti Technique for the Treatment of Congenital Club foot at OPD in Rajshahi Medical College Hospital
}

\author{
D M Sohel ${ }^{1}$, A Asif Iqbal ${ }^{2}$, P Dutta ${ }^{3}$, B K Dam ${ }^{4}$, S Ahmed $^{5}$, T Yasmin $^{6}$
}

\begin{abstract}
Congenital club foot is the most common congenital deformity of the foot. Its characteristics presentation of equinus, varus, adductus and cavus deformities makes it easily recognizable. The estimated ratio is 1 or $\mathbf{2}$ per 1000 live births.It is a complex deformity that is difficult to correct. The goal when treating idiopathic clubfoot is achieve a foot with appearance and function that are nearly normal. There various types of treatment options for correction of the foot. (1) conservative, (2) surgery. The most frequently used surgical approach is posterio medial release which has many variation. However, long term follow up studies showed that the results of surgical treatment are disappointing. Ignacio Ponseti MD is the man who changed the previous treatment plan of club foot. Ponseti developed his method of club foot manipulation based on his understanding of normal anatomy of subtalar joint. In OPD of Rajshahi Medical College Hospital we studied 262 patients with congenital club foot, in which 188 were male and 74 were female. Total number of tenotomy undertaken 172, patients using braces 200 . In our short term follow up our result is encouraging.
\end{abstract}

TAJ 2010; 23(2): 26-29

\section{Introduction}

Congenital club foot or congenital talepes equinovarus (CTEV) is the most common congenital deformity seen in orthopaedics practice. It is a complex deformity that is difficult to correct. The deformity has four components Equinus, Varus, Adductus and Cavus., ${ }^{1,2,3}$ This condition is twice as common in males than females. ${ }^{2,3}$ Estimated ratio is 1 or $2: 1000$ live births. $^{2.3}$ The goal of treatment is to reduce or eliminate these four deformities, so that the patient has a functional, pain free, plantigrade foot with good mobility and does not needed to wear modified shoes.
John Herzenberg stated 3 dimensional CT for club foot deformities, these are comprises of Navicular bone is severely medially displaced, Talus is severe planterflexed, its neck is medially and planterly deflected and head is wedge shaped. Body of the talus externally rotated with in the ankle mortes. Calcaneus is adducted and inverted under the talus, Calcaneo cuboid joint is distorted and cuboid is underneath of navicular bone., ${ }^{2,3,4}$

There are various types of treatment options for clubfoot. One is conservative and another surgery. In conservative method: passive stretching with splintage, serial plaster with assessment, Ponseti method. In surgery there are various types of

1 Registrar, Department of Ortho-Surgery, Rajshahi Medical College and Hospital, Rajshahi.

2 Assistant Professor, Reconstructive Surgery, Rajshahi Medical College, Rajshahi.

3 Assistant Professor, Department of Pediatrics, Rajshahi Medical College, Rajshahi.

4 Professor \& Head, Department of Ortho-Surgery, Rajshahi Medical College, Rajshahi.

5 Associate Professor, Department of Ortho-Surgery, Rajshahi Medical College, Rajshahi.

6 Ponseti Practitioner, Clinic Manager, Rajshahi. 
operative procedures are performed. Soft tissue procedure: PMR with Close Steindler. Bony procedure: Osteotomy, Decancellation of specific bone specially cuboid bone, Triple arthrodesis. ${ }^{5}$

Ignacio Ponseti, MD is the man who changed the previous treatment plan of club foot. Ponseti developed his method of clubfoot manipulation based on his understanding of normal anatomy of the subtalar joint. He views the club foot deformity as an exaggerated position of the ankle and subtalar joint. Fore foot is maximum varus, equinus and inversion undoing the positions means reversing the direction of the subtalar and ankle joints into maximum valgus, dorsiflexion and eversion. All Ponseti's maneuvers are based on taking advantage of the natural movements of the subtalar and ankle joints.

The ponseti technique is effective because it takes advantage of the kinematics of the subtalar joint. The subtalar joint is not a fixed axis but an axis that changes with foot structure and position. Therefore, subtalar joint movements (Pronation/supination) around a moving axis generate complex, continuously altering positions. $^{6}$

In Ponseti technique manipulation and serial application of plaster cast supported by limited operative intervention (percutaneous tenotomy) yielded satisfactory functional results about $94 \%$ of the foot in ponseti clinic by Ponseti technique.

In some centre early and even primary operative treatment is practised but often some failure and complications are common. Long term follow up studies have shown that the results of surgical treatment are disappointing. Increasing foot pain, weakness, and stiffness often lead to premature arthritis and disability of the foot.

Some people believe as Ponseti does that 'the successful non operative or limited operative treated foot is much better than the successful surgically treated foot'.

\section{Material and Methods}

- Type of study: Prospective clinical study.

- Place of study: Out patient Department in Rajshahi Medical College Hospital.
- Duration of study: 4 March 2010 to 30 June 2011.

- Study population: Patients with congenital club foot attending at OPD of RMCH.

- Age group: 0-3yrs of age.

- Sex group: Both male and female.

- Method: Ponseti technique.

- Sample size: 262 patients.

\section{Method}

The ponseti method consist of two phases. (1) Treatment phase (2) Maintenance phase.

\section{(1) Treatment phase}

Should begin as early as possible. Gentle manipulation and casting are done weekly. Cast holds the foot in the corrected position, allowing it to reshape gradually. Usually four to six cast required to correct the alignment of foot and ankle fully. At the time of the final cast most infants $(\geq 70 \%)$ require percutaneous achilles tenotomy to gain adequate lengthening of the achilles tendon.

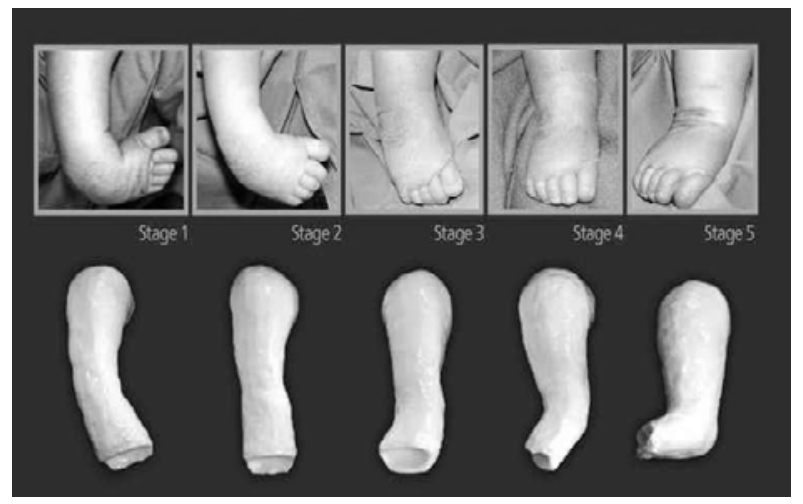

Photograph of gradual correction clubfoot by ponseti technique with serial plaster cast (Courtesy: Clubfoot: Ponseti Management, GLOBAL HELP)

\section{(2) Maintenance phase}

In Maintenance phase it is maintained by Danis Browne splint. Splinting started after the removal of final cast or tenatomy cast 3 weeks after tenatomy. The splint consist of shoes to a bar in a position of $70^{\circ}$ of external rotation and $15^{\circ}$ of dorsiflexion. The distance between shoes is set at about 1 inch wider than the width of the infants' shoulder. Splint wear initial 3 months for at least 23 hours of a day and then 12 to 14 hours of a day up to 3 to 4 years of the patient. 


\section{Results}

Total number of patient 262. Age of the patients ranges from 07 days to 36 months of them 147 patients were under 01 year of age. Both male \& female were studied, bilateral: 121, unilateral: 141. The number of plaster ranges from 4- above 6 plaster (4 plaster 167 patients, 5 plaster 38 patients and above 6 plaster 57 patients), patients were treated along with tenotomy were 172 feet. The result is regarded as Dr. Shafiq Pirani score by 6 clinical sign which is either $0,0.5$ and 1 . Final results were excellent with pirani score-0, $92(\mathrm{Lt})$ ,109 (Rt) feet. Results were good with pirani score-0.5, 28 (Lt), 36 (Rt) feet and rest of the feet were satisfactory with pirani score-1.
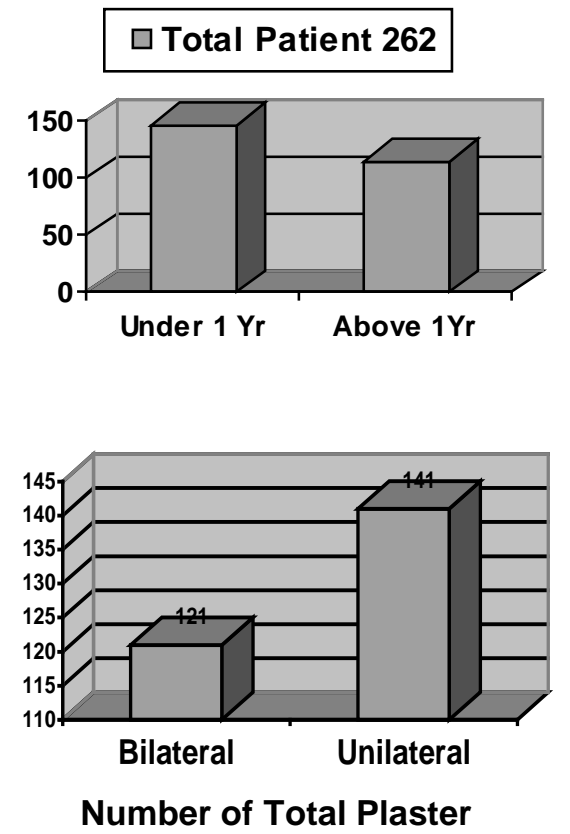

\section{$\square$ Plaster $\square 5$ Plaster $\square$ Abo}

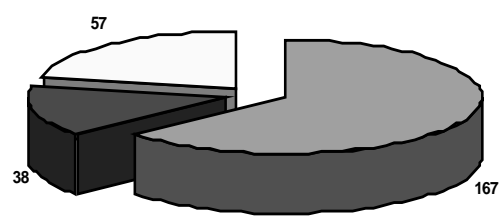

\section{Excellent with Pirani Score 0}

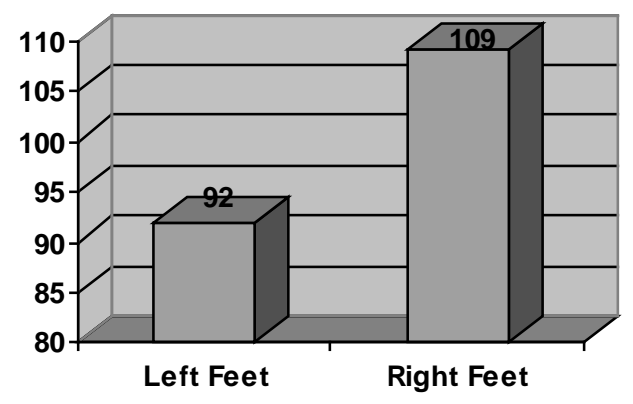

Good With Pirani Score 0.5

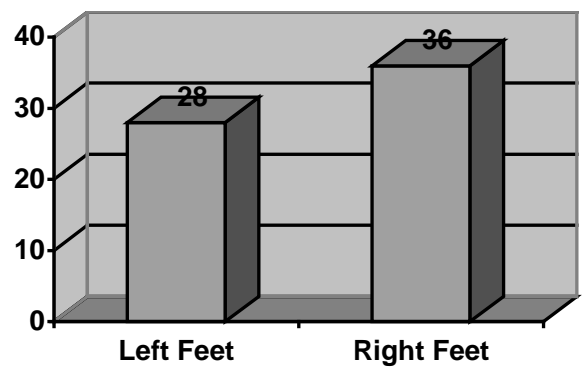

\section{Discussion}

The congenital club foot is the most common congenital deformity of foot. It is complex deformity comprises of equinus, varus, adductus and cavus which is difficult to correct. The goal of treatment reduced or eliminated these four deformities, so that the patient has a functional pain free plantigarde foot with good mobility and does not need to wear modified shoes. The conventional treatment of club foot is serial plaster, two weekly or three weekly up to 7 or eight plaster or surgery (PMR) in different methods and approaches. All the procedure have different complications like inadequate correction, stiffness, weak, severe scar and painful foot. On the other hand Ponseti technique yielded satisfactory anatomical functional result, with simple, effective, minimally invasive, inexpensive and ideally suited for all countries and culture. The Ponseti technique is practiced in many countries of the world. All the parents are satisfied about the children's corrected foot with Ponseti technique.

The difficult part of the Ponseti technique is the maintenance phase. The baby may feel 
uncomfortable at first when trying to alternatively kick the legs. However, the baby soon learns to kick both legs simultaneously and feels comfortable. The parents reported that initial 2-3days were the critical period, during which patient were restless and tried to remove the splint, after that period patients were adjusted with the splint.

\section{Conclusion}

The Ponseti method is the most effective, safest and cost effective treatment for idiopathic club foot with excellent result and negligible morbidity. It has been successful through out the world in all cultures and level national development. In our short term follow up our result is encouraging.

\section{References}

1. Md. Jahangir Alam et al 'The ponseti technique for the treatment of congenital clubfoot' Journal of Bangladesh Orthopaedic Society January 2009, vol-24, No.1, pp28-32

2. Louis Solomon et al 'Appley's System of Orthopaedics and Fractures' 8th edition Holder Arnold, London 2001 pp488-491.

3. Wallace B. Lehman MD 'The clubfoot first edition. JB Lippincott Co. Toronto 1980 pp2-103.

4. Herzenberg JE, Radler C, Bor N. Ponseti versus traditional methods of casting for idiopathic clubfoot. J Pediatr Orthop2002;22(4): 517-521

5. Canale, ST 2004, Compell's operative orthopedics, 10th edn, Mosby, London, pp988-1006

6. Spoor CW, Huson A, van Langelaan EJ. Comment on 'A biomechanical analysis of the talocalcaneal joint-in vitro. J Biomech 1988; 21 (11):993-994. 